Research in Astron. Astrophys. Vol.0 (200x) No.0, 000-000

http://www.raa-journal.org http://www.iop.org/journals/raa

Research in

Astronomy and

Astrophysics

\title{
Quark Nova Model for Fast Radio Bursts
}

\author{
Zachary Shand, Amir Ouyed, Nico Koning and Rachid Ouyed \\ Department of Physics and Astronomy, University of Calgary, 2500 University Drive NW, \\ Calgary AB T2N 1N4, Canada rouyed@ucalgary.ca
}

\begin{abstract}
FRBs are puzzling, millisecond, energetic radio transients with no discernible source; observations show no counterparts in other frequency bands. The birth of a quark star from a parent neutron star experiencing a quark nova - previously thought undetectable when born in isolation - provides a natural explanation for the emission characteristics of FRBs. The generation of unstable r-process elements in the quark nova ejecta provides millisecond exponential injection of electrons into the surrounding strong magnetic field at the parent neutron star's light cylinder via $\beta$-decay. This radio synchrotron emission has a total duration of hundreds of milliseconds and matches the observed spectrum while reducing the inferred dispersion measure by approximately $200 \mathrm{~cm}^{-3} \mathrm{pc}$. The model allows indirect measurement of neutron star magnetic fields and periods in addition to providing astronomical measurements of $\beta$-decay chains of unstable neutron rich nuclei. Using this model, we can calculate expected FRB average energies $(\sim$ $10^{41}$ ergs), spectra shapes and provide a theoretical framework for determining distances.
\end{abstract}

Key words: stars: neutron - nuclear reactions, nucleosynthesis, abundances radiation mechanisms: general — radio continuum: general

\section{INTRODUCTION}

The observation of fast radio bursts (FRBs) has created an interesting puzzle for astronomers. Explaining the origin of these millisecond bursts has proven difficult because no progenitor has been discovered and there is no detectable emission in other wavelengths. Many possible explanations for these bursts have been put forward: blitzars (Falcke \& Rezzolla 2014), neutron star mergers (Totani 2013), magnetars (Popov \& Postnov 2007, 2013; Lyubarsky 2014; Pen \& Connor 2015), asteroid collisions with neutron stars (Geng \& Huang 2015), and dark matter induced collapse of neutron stars (Fuller \& Ott 2015), to name a few; however, none so far have been able to explain the many puzzling features of the emission: the signal dispersion, energetics, polarization, galactic rate, emission frequency, and lack of (visible) companion/source object.

Direct observational evidence for quark stars is difficult to accrue due to some of their similarities to neutron stars (e.g. compactness, high magnetic field, low luminosity). In our model, FRBs signal the birth of a quark star and as more FRB observations become available, they may be able to provide evidence for the existence of quark stars. Other theoretical observational signatures of quark stars include: gravitational waves from quark star oscillation modes (Gondek-Rosińska et al. 2003; Flores \& Lugones 2014); gravitational waves from a strangequark planet and quark star binary system (Geng et al. 2015); equation of state constraints of the mass-radius relationship (Drago et al. 2015) and high-energy emission directly from the quark star in X-ray and gamma ray emission (Ouyed et al. 2011). 
The Quark Nova (hereafter QN) model has many ingredients which prove useful when trying to explain FRBs. Firstly, the parent neutron star has a strong magnetic field which can generate radio synchrotron emission. Secondly, the neutron rich ejecta of the $\mathrm{QN}$ has a unique mechanism for producing these electrons via $\beta$-decay of nuclei following r-process nucleosynthesis in the QN ejecta (Jaikumar et al. 2007; Charignon et al. 2011; Kostka et al. 2014b). Our model makes use of $\beta$-decay of nuclei in the ejecta of a QN to produce radio synchrotron emission. The exponential decay inherent to the nuclear decay explains the millisecond pulses observed in a way that no other model so far can.

As an isolated, old neutron star spins down the central density increases. Neutron star spin-down, which increases the core density leading to quark deconfinement, in conjunction with accretion and s-quark seeding can trigger an explosive phase transition (Ouyed et al. 2002; Staff et al. 2006; Ouyed et al. 2013). This explosion ejects a dense layer of neutrons which produce unstable heavy nuclei (Keränen et al. 2005; Ouyed \& Leahy 2009). These unstable nuclei decay and become observable at the light cylinder (hereafter LC) where they emit synchrotron emission due to the strong magnetic field. (For a $10 \mathrm{~s}$ period and $10^{12} \mathrm{G}$ parent neutron star this corresponds to $B \sim 300 \mathrm{G}$ at the LC.) This generates a unique geometry and field configuration where our emission occurs at the intersection of a cylinder and sphere in space traced out by the ejecta as it passes through the LC.

We expect FRBs to be associated with old, isolated neutron stars and not young, rapidly rotating neutron stars. Neutron stars born with short periods quickly spin down and increase their central densities and, when these neutron stars are born with sufficient mass, deconfinement densities are reached very quickly (within days to weeks). Instead of emitting as an FRB, these QN will interact with the supernova ejecta leading to either a super-luminous supernova (Kostka et al. 2014a), or a double-humped supernova (Ouyed et al. 2013). This interaction with the surrounding material is expected to either disrupt the FRB emission, or bury the signal in the surrounding dense ejecta. The remaining neutron stars which are born massive and with high periods will take much longer (millions of years) to achieve the required critical core density. As such, we expect a continuum of FRB progenitors with a range of periods and magnetic fields to be emitting at various frequencies and luminosities.

Two important consequences of this scenario are: 1) an extended time spectra as the sphere sweeps across higher and higher latitudes of the LC and 2) an exponential time profile of millisecond duration from decay of $\beta$-decaying nuclei. As is demonstrated later (in Fig. 6), these qualities match observations of FRBs and allow us to provide testable predictions for distances, energies, rates and bandwidth of future FRB measurements which we can link to old, isolated neutron star populations and nuclear rates.

\section{MODEL OVERVIEW}

As a neutron star ages, spin-down increases its central density. For massive enough neutron stars, this can cause a phase transition birthing a quark star. The exploding neutron star ejects - at relativistic speeds - the outer layers of the neutron rich crust. The ejecta is converted to unstable r-process material in a fraction of a second and reaches a stationary r-process distribution which is maintained by the high-density of the ejecta and the latent emission of neutrons from fission (fission recycling). This distribution of unstable nuclei, which normally would decay within seconds, can continue to exist while the density is high and fissionable elements are able to release neutrons back into the system. This stationary distribution will be disrupted by decompression (or other disruption) of the QN ejecta as it passes through the LC of the surrounding parent neutron star magnetic field.

Once the ejecta is beyond the current sheets of the LC, the QN ejecta will no longer support a stationary r-process distribution and electromagnetic radiation from the ejecta will no longer 


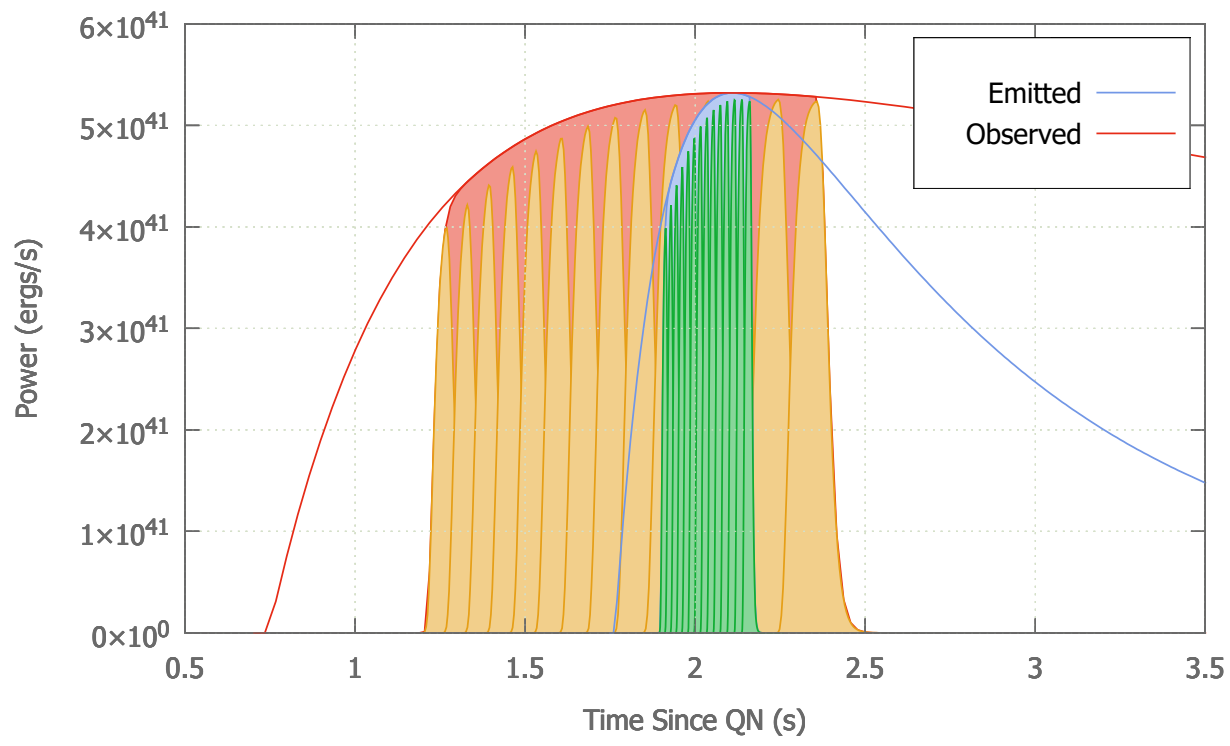

Fig. 1 Light curve of the FRB emission at the source (blue) and the observed emission with dispersion smearing (red). The filled portion of the curves indicates the viewable emission for bandwidth limited observation in the range of 1175-1525 MHz. The green and orange pulses are $25 \mathrm{MHz}$ sub-bands of the signal which exhibit the characteristic millisecond pulse duration.

be attenuated by the LC plasma, becoming visible ${ }^{1}$. The nuclei will decay via a rapid series of $\beta$-decays. These generated electrons are emitted with $\mathrm{MeV}$ energies in a strong magnetic field producing radio synchrotron emission. The frequency and power of this emission depends, of course, on the magnetic field strength at the LC where the QN ejecta crosses it. As a result, the emission spectrum is controlled by the geometry of the spherical intersection of the ejecta with the LC and allows inference of the progenitor neutron star's period and magnetic field.

A distinguishing detail in our model is the duration of the emission, which has a duration of seconds instead of milliseconds. The signal is a continuous series of synchrotron emissions from bunches of electrons generated from $\beta$-decay, which produce coherent, millisecond duration pulses at each frequency over several seconds (or hundreds of milliseconds for a bandwidth limited observation), as can be seen in Fig. 1. This is in apparent contrast with observations, which indicate a total emission duration of milliseconds. This discrepancy in the FRB duration stems from the de-dispersion process which stacks all frequency channels to a common initial time (as is done in pulsar de-dispersion and signal folding). As such, according to our model, the FRB emits a series of coherent, self-similar signals at different frequencies which have been erroneously stacked as a time synchronized pulse: the total duration of the FRB is actually several seconds long and the observed signal duration is still dominated by cold plasma dispersion, as is demonstrated by the red/orange signal in Fig. 1.

The model comprises a combination of relatively standard physics phenomena: $\beta$-decay, magnetic field geometry and synchrotron emission. In composite, they generate a unique emission mechanism reminiscent of FRBs. The following sections (Sections 2.1 to 2.4) will be spent outlining each component of the model in greater detail and setting out the relevant equations

1 The current sheets at the light cylinder produce a Faraday cage effect which shields the emission of $\beta$-decay occurring before the r-process material crosses the LC. 
necessary to generate the equations which describe the synchrotron emission spectra of the r-process material generated in the QN ejecta (see Section 3).

\subsection{R-process Elements}

The formation of heavy elements by the r-process is one of the predictions of the QN model (Jaikumar et al. 2007; Charignon et al. 2011; Kostka et al. 2014b). Due to large number of free neutrons delivered by the ejected neutron star crust, it is possible for heavy fissile nuclei to be created during the r-process. R-process calculations show that superheavy $(Z>92)$ fissile elements can be produced during the neutron captures. This leads to fission recycling during the r-process (Kostka et al. 2014b). Each fission event ejects (or releases) several ( $\sim 5-10)$ neutrons back into the r-processing system. (This is due to neutron evaporation during asymmetric fission and is same mechanism present in nuclear reactors.) Because of this, once these fissionable elements are produced, we are guaranteed to have free neutrons continually resupplied to the system which recombine into heavier elements which will in turn fission again and create a feedback loop in the r-process inside the QN ejecta. This allows for continued neutron captures and "r-processing" much longer than traditionally expected (see Fig. 2). These unstable isotopes are then able to supply us with the electrons which will decay in coherent bunches over different magnetic fields to produce the final aggregate emission spectrum.

\subsubsection{R-process Rates}

The basic r-process mechanism operates as a competition between neutron capture reactions $(\mathrm{n}, \gamma)$ and its inverse photo-dissociation $(\gamma, \mathrm{n})$ with the much slower $\beta$-decays slowly building up each successive isotope. This picture holds true for most of the r-process; however, as the gas expands and cools, the photo-dissociation rates fall off very quickly with temperature. The neutron-capture rates, on the other hand, do not have as strong a temperature dependence and are mainly a function of neutron density (see Fig. 3).

For an initially dense ejecta (which is of course the case for a neutron star crust $(\rho \sim$ $10^{14} \mathrm{~g} \mathrm{~cm}^{-3}$ ) this means that neutron captures can continue at low temperatures even after significant initial decompression. Instead of competing with photo-dissociations, they will begin to compete with $\beta$-decays instead as shown in Fig 3. At this point, simulations show that the isotopic distribution enters an approximate steady state (possible because of the fission recycling) which can continue until the density and subsequently the neutron capture rates lower sufficiently. The rates shown here come from Möller et al. (2003) and the BRUSLIB database (Goriely et al. 2008, 2009). These rates are all theoretical due to the difficulty of measuring them in the lab (Krücken 2011) and presents a way of observationally constraining $\beta$-decay rates by measuring synchrotron radiation from theses decay chains.

\subsubsection{Decay Chain of Unstable Isotopes}

For elements lighter than the actinides, $\beta$-decay (or electron capture) is the dominant decay mode available to them. These decay chains can be described by coupled differential equations which have a simple analytic solution known as the Bateman equations (Krane \& Halliday 1988):

$$
\begin{gathered}
\mathscr{A}_{n}=N_{0} \sum_{i=1}^{n} c_{i} e^{-\lambda_{i} t}, \\
c_{m}=\frac{\prod_{i=1}^{n} \lambda_{i}}{\prod_{i=1}^{n} \prime\left(\lambda_{i}-\lambda_{m}\right)},
\end{gathered}
$$

where the prime indicates that the lower product excludes the term where $\mathrm{i}=\mathrm{m}$. This equation gives the activity (number of events per unit time) of a decay chain. For a purely $\beta$-decay chain 


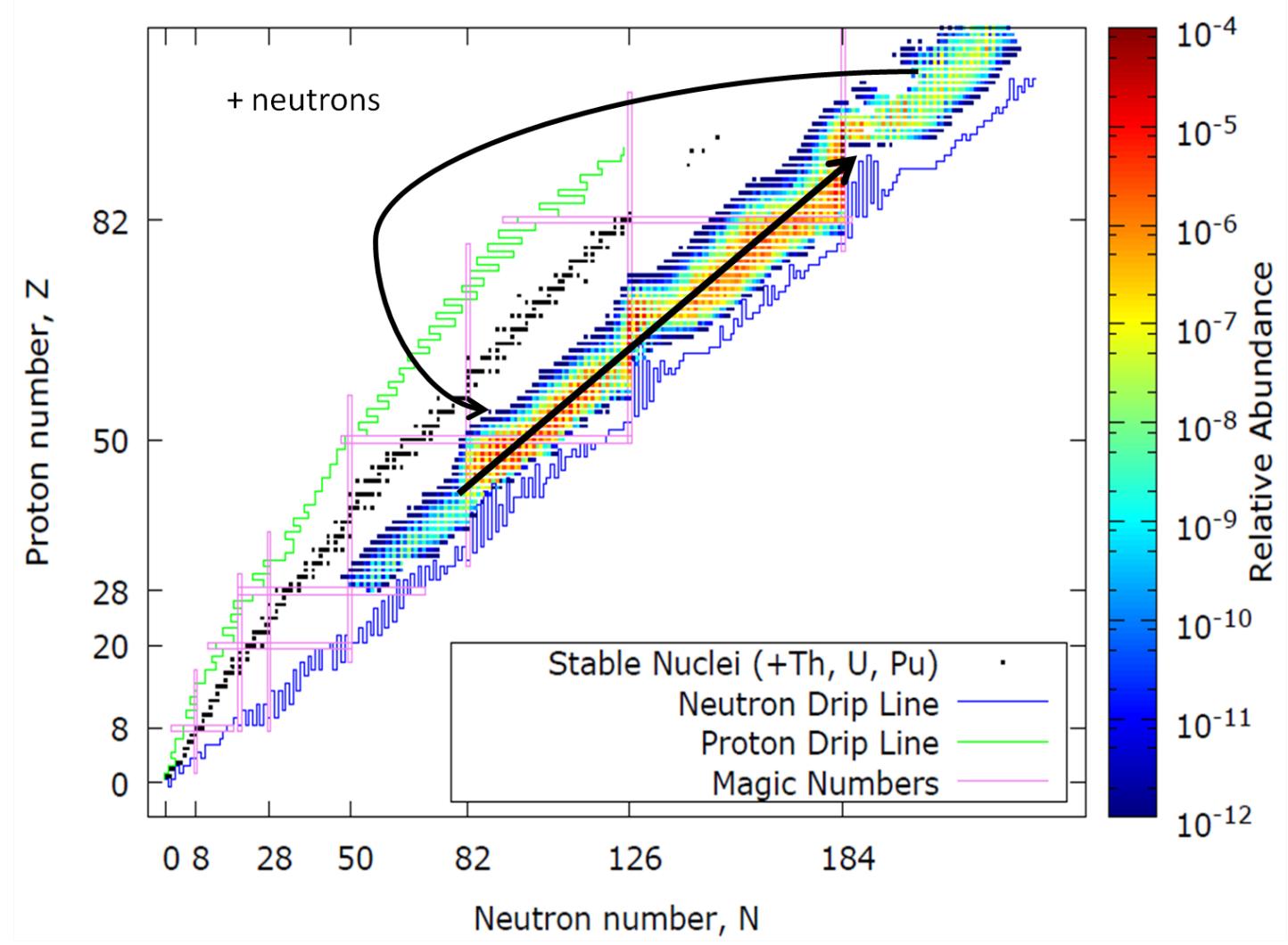

Fig. 2 Stationary distribution of the r-process near the end of the r-process. The neutron source becomes largely depleted and no major changes to the distribution occur; however, fissionable nuclei emit neutrons and create a cyclic recycling process which can maintain a distribution of unstable nuclei for several seconds $(>10 \mathrm{~s})$ in contrast to the milliseconds required for generation of r-process material. The distribution is computed using r-Java 2.0 as in Kostka et al. (2014b).

this gives the rate at which free electrons are produced by nuclei. As can be seen in Fig. 4, once $\beta$-decay dominates, the unstable nuclei will decay roughly exponentially and have a short burst of activity that will emit synchrotron radiation if subject to an external magnetic field. This naturally gives us an exponential fall-off time once neutron captures stop, which as discussed above, can be much later in the expansion than traditionally expected in the r-process due to the high density of the ejected material (which is abruptly triggered by decompression across the LC). Once emitted, the electrons will recombine with the surrounding weakly ionized plasma which should prompty emit in the $\mathrm{keV}$ range which could be visible if the plasma is diffuse enough; however, at much lower total emission power than the FRB burst itself (likely making it undetectable).

\subsection{Geometry and Field Configuration}

Assuming that the emission is triggered at the LC, we need to define a coordinate system with which to describe emission time and magnetic field strength (see Fig. 5). The LC is simply 

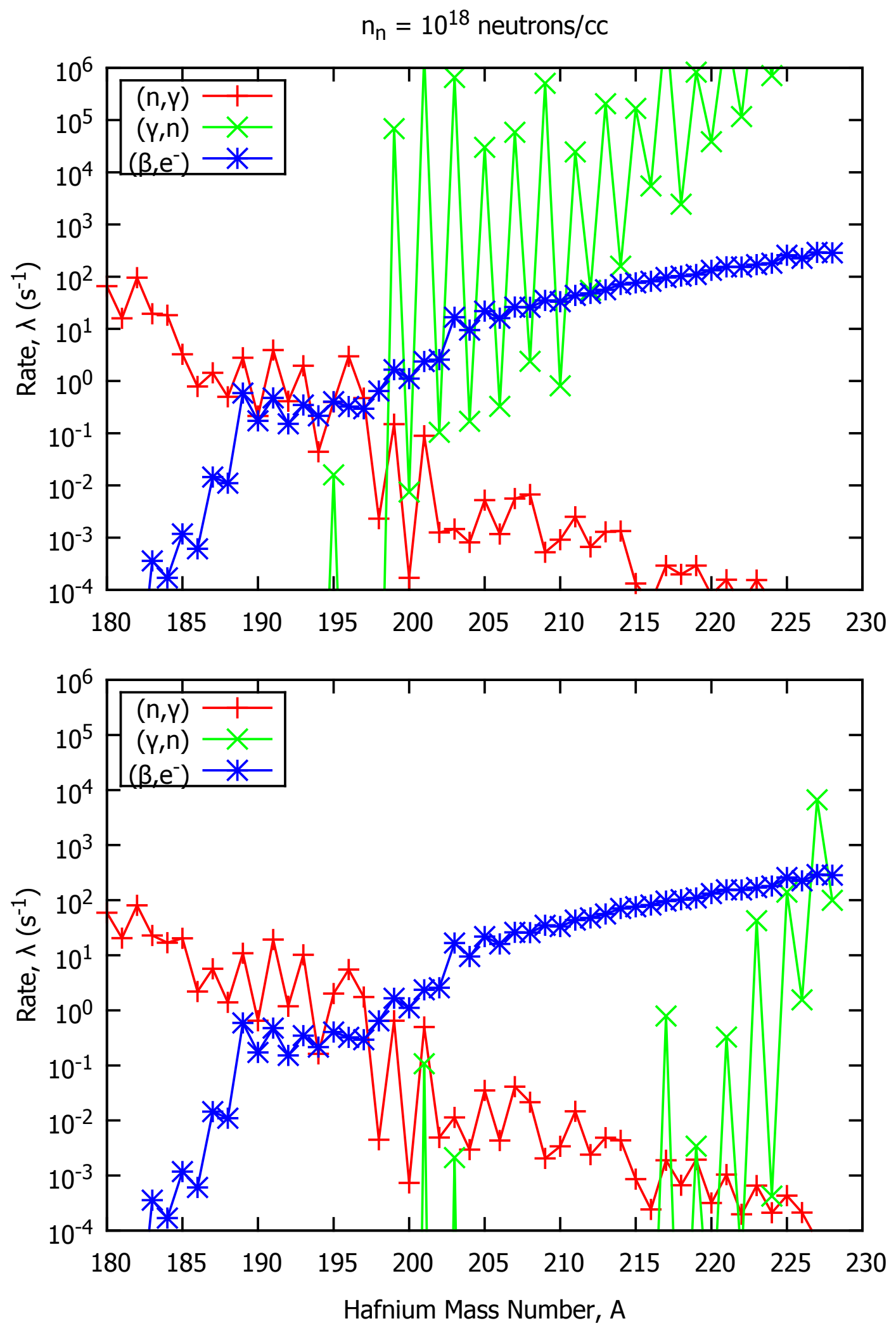

Fig. 3 Relevant temperature dependent rates for hafnium show different regimes in the r-process. The neutron density is $10^{18} \mathrm{~cm}^{-3}$ and the temperatures are $1.0 \times 10^{9} \mathrm{~K}$ (top) and $0.5 \times 10^{9} \mathrm{~K}$ (bottom). 

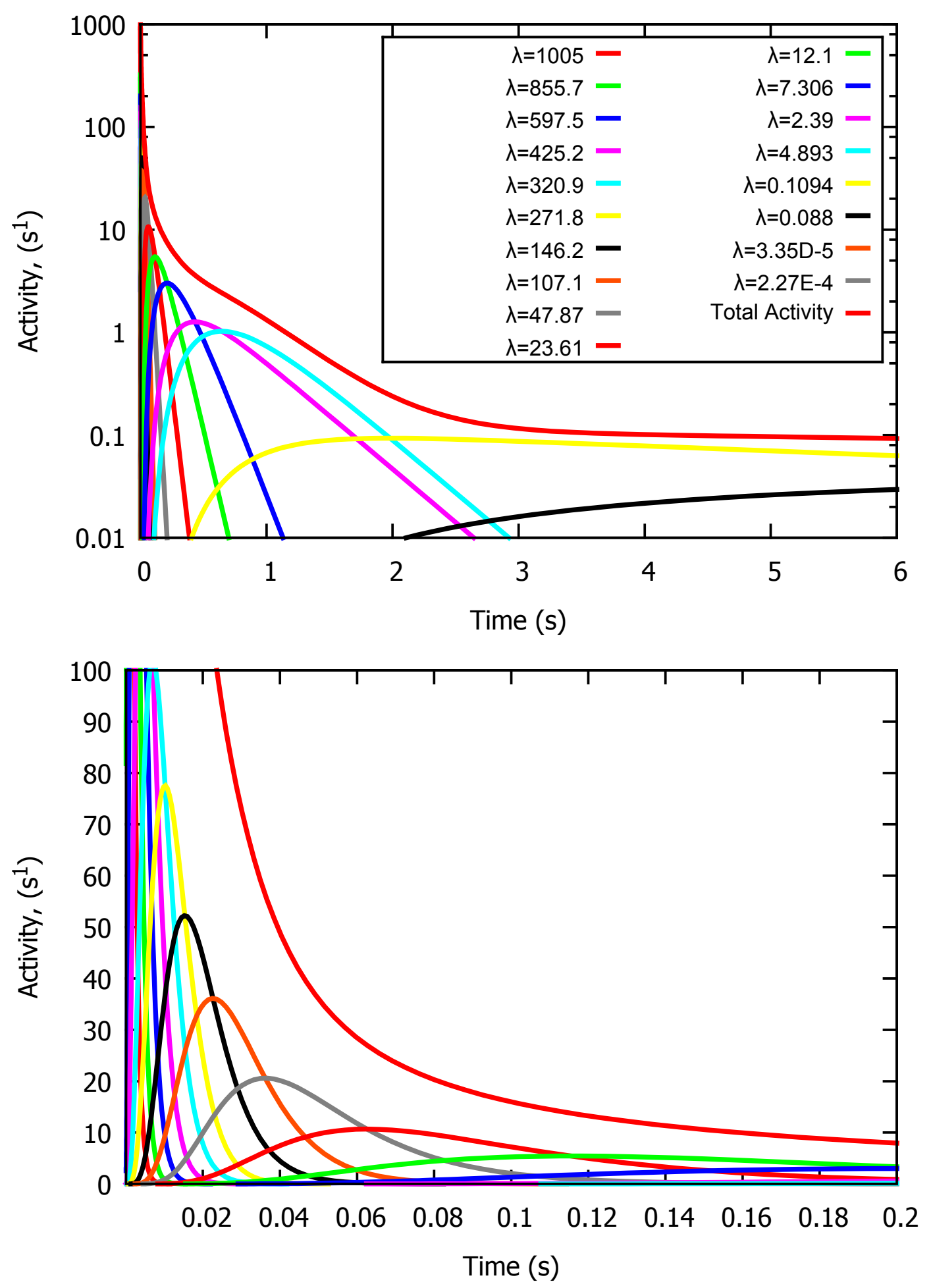

Fig. 4 Solution of the Bateman equation for the $A=156 \beta$-decay chain beginning near the drip-line. Once the neutron decays stop competing with $\beta$-decays at the end of the r-process (when the density falls sufficiently), the activity of the material will $\beta$-decay with this characteristic exponential behaviour. The top panel is logarithmic in the activity (y-axis) and the bottom panel is the very early portion of the above panel without the logarithmic y-axis. 


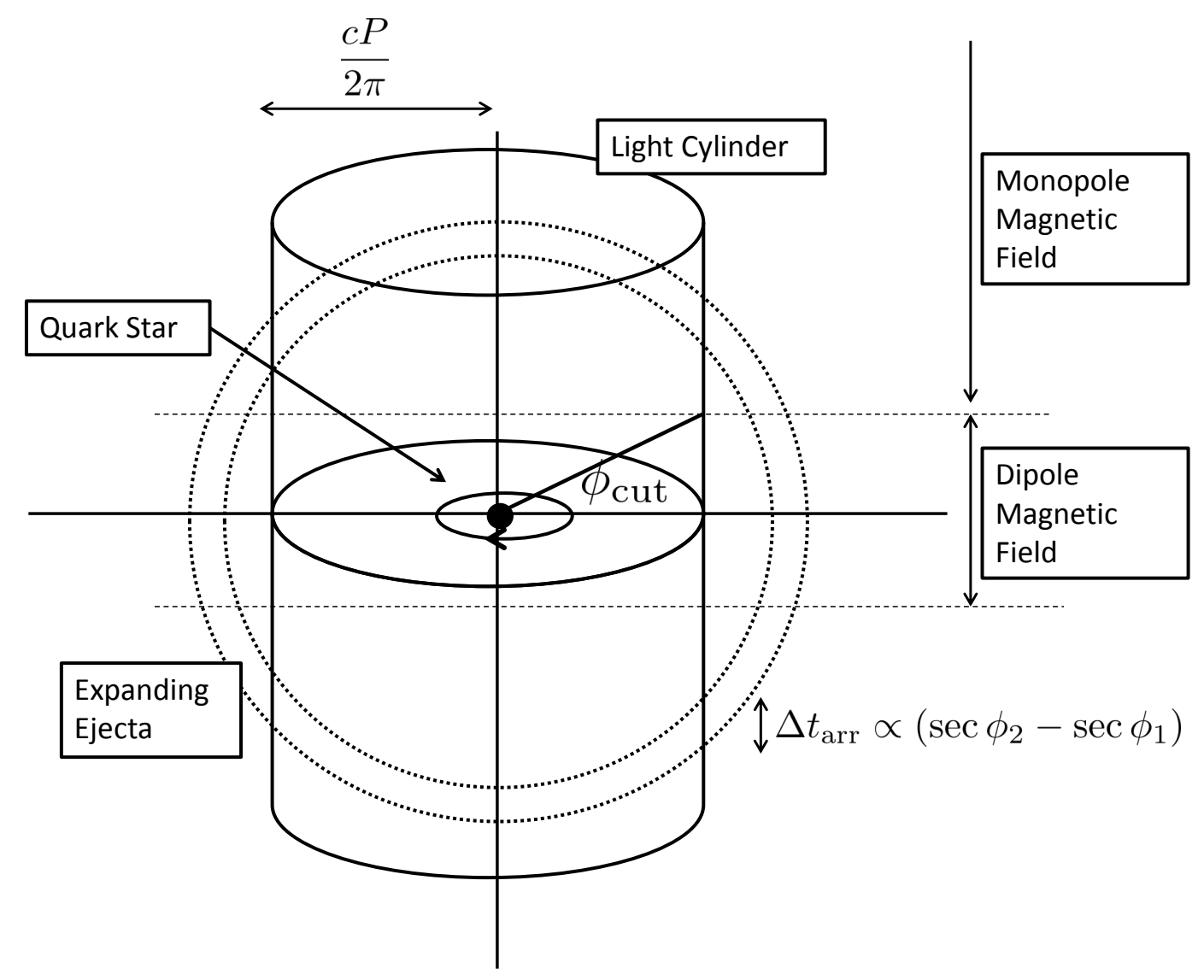

Fig. 5 Diagram of outlining geometry of the model. The latitiude angle angle is specified relative to the quark star location and depicts the angle at which the spherical QN ejecta intersects with the LC. The figure depicts two intersections of the ejcta with the cylinder at two different latitude angles separated by the arrival time of the two latitue angles.

defined as the point where a co-rotating magnetic field would be rotating at the speed of light. The magnetic field configuration has a breakpoint in it where the magnetic field lines no longer reconnect and fall off as a mono-polar magnetic field. For a LC radius defined by: $r_{L C}=\frac{c P}{2 \pi}$, where $\mathrm{c}$ is the speed of light and $\mathrm{P}$ is the period, the magnetic field strength at the LC at latitude, $\phi$, is given by:

$$
B=B_{0}\left(\frac{r_{0} \cos \phi}{r_{L C}}\right)^{a},\left\{\begin{array}{cc}
a=3 & 0^{\circ} \leq \phi<30^{\circ} \\
a=2 & 30^{\circ} \leq \phi<90^{\circ} .
\end{array}\right.
$$

This will cause radiation in the equatorial plane, where the dipolar field configuration exists, to be a factor of $c$ weaker ( $c^{2}$ for power).

For a relativistic, spherical shell this also provides a straightforward description for the arrival time of the material at the LC:

$$
t_{\text {arr }}=t-\frac{P}{2 \pi \beta} \sec \phi
$$


which provides a time delay between emission at different latitude angles.

\subsection{Light Cylinder Details and Magnetic Field}

The magnetic field configuration outside due to the LC will transition to a monopole magnetic field for an aligned rotator (Michel 1973; Contopoulos et al. 1999) or will have more complicated shape for an unaligned rotator (Spitkovsky 2006). As the QN ejecta passes through this region, the magnetic field will be disrupted and the details of the dynamics would be complicated to simulate; however, the disruption of the currents and the change of the magnetic field are assumed to cause a disruption of the QN ejecta. The details of the expansion across the LC are beyond the scope of this paper; however, as the ejecta passes through the boundary, there will be decompression of the material as the magnetic field distribution changes and the currents at the LC will interact with the matter and be disrupted. Since the LC inhibits emission of electromagnetic radiation across the LC (Jessner et al. 2000), this physical disruption can help to explain the sudden rise of the emission of the FRBs.

Both the neutron star and quark star will have a LC and so there is some possible ambiguity in what magnetic field will be present at the LC when the ejecta crosses it. Since QN ejecta is highly relativistic $(v \sim 0.99 c)$ we expect it to reach the LC of the parent neutron star before there is time for the quark star to fully rearrange the magnetic field around it which occurs much slower (Ouyed et al. 2006). This can allow inference of properties of the neutron star population which undergo quark-nova and emit these FRBs.

\subsection{Synchrotron Emission}

The emitted electrons are assumed to have a constant $\gamma$ across all decaying elements. This is done both to simplify the model and also due to a lack of nuclear data of $\beta$-decay electron energy distributions. If we look at the flux density, this factor is suppressed and so the assumption ends up not factoring into comparisons for flux density (see Fig. 9 for expanded form of the equations).

The synchrotron frequency and power per electron are given by:

$$
\begin{gathered}
\nu_{\text {sync }}=\kappa_{\nu} \gamma^{2} B, \\
P_{e}=\kappa_{p} \gamma_{\perp}^{2} B^{2},
\end{gathered}
$$

where $\kappa_{p}=1.6 \times 10^{-15} \mathrm{ergs} \mathrm{s}^{-1} \mathrm{G}^{-2}$ and $\kappa_{\nu}=1.2 \times 10^{6} \mathrm{~Hz} \mathrm{G}^{-1}$ are constants (Lang 1999). For simplicity, we have used the synchrotron peak frequency in place of the sharply peaked complete distribution.

If we assume the electrons only emit for a short time (either due to recombination or dissipative processes) then the number of synchrotron electrons is the same as the activity, $\mathscr{A}$. The activity is, of course, related to the decay constants of the unstable neutron-rich r-process nuclei:

$$
N_{\text {total }}(t)=\sum_{i}^{\text {chain }} \frac{\mathscr{A}_{i}}{\lambda_{i}}
$$

We write this explicitly to emphasize that there are many nuclear species present at the end of the r-process and there are many decay rates simultaneously contributing. This makes it difficult to constrain individual $\beta$-decay rates, but can help to constrain nuclear theory models (not unlike the r-process).

\subsection{Summary of Model Components}

Our model involves many individual components as outlined above in sections 2.1-2.4 and so we will shortly summarize how each qualitatively affects the emitted spectra. The r-process 
and fission recycling generate the electrons required for synchrotron emission and light cylinder provides a valve-like disruption and a monopole magnetic field. The spectrum is affected by the characteristics of the geometry and nuclear decay that exists in a QN. The exponential decay generates an exponential pulse at each particular emission frequency; whereas, the period of the neutron star affects magnetic field strength that the nuclei see as they cross the LC. In this simple model, the QN ejecta emits at decreasing energies and frequencies as the latitude of intersection increases and the exact bandwidth of the emission is determined by the parent neutron star's period and and LC field. In Fig. 9 we show a total expression for the emitted spectrum.

\section{TOTAL POWER AND SPECTRUM}

The total number of nuclei and by extension electron emitters at a particular latitude is expressed as:

$$
N_{e}=\sum_{i}\left(\frac{\mathscr{A}_{i}}{\lambda_{i}}\right) \int_{\phi_{1}}^{\phi} \cos \phi^{\prime} d \phi^{\prime}=\sum_{i}\left(\frac{\mathscr{A}_{i}}{\lambda_{i}}\right)\left(\sin \phi-\sin \phi_{1}\right) .
$$

The number of emitting electrons is then conveniently defined as a fraction of the total activity of the ejecta which is related to the total number of initial radioactive isotopes as defined in Eq. 1. This is simply the total number of isotopes in the quark nova ejecta and is given by:

$$
N_{0}=\frac{m_{\mathrm{QN}, \text { ejecta }}}{A_{\text {avg }} m_{H}}
$$

The power then decays exponentially in time according to the activity of the nuclear species in the ejecta as it passes through the LC.

In order to determine the total power over the entire area we integrate the polar angle and latitude while keeping in mind that there is a time delay associated with the arrival of each latitude angle, $\phi$ due to the intersection of the spherical shock wave with the LC (giving us the Heaviside theta function, $\Theta$ ).

We can then construct an expression for the instantaneous power and spectral flux density as follows:

$$
\begin{aligned}
& \mathscr{P}=\Theta(t) P_{e} N_{e}, \\
& \mathscr{S}=\frac{\Theta(t) P_{e} N_{e}}{\nu},
\end{aligned}
$$

which are all evaluated at the arrival time (Eq. 4) which has an angular dependence. These expressions can then can be integrated to get the total power or spectral flux density:

$$
\begin{aligned}
& P(t)=\oiint_{S(\theta, \phi)} d P=\oiint_{S(\theta, \phi)}\left[\frac{\partial \mathscr{P}}{\partial \phi}\right] d \Omega, \\
& S(t)=\oiint_{S(\theta, \phi)} d S=\oiint_{S(\theta, \phi)}\left[\frac{\partial \mathscr{S}}{\partial \phi}\right] d \Omega,
\end{aligned}
$$

which are both very unfriendly looking integrands; however, they both only have functional dependence on latitude angle, $\phi$.

It is worth noting here that these integrals are evaluated at the source (in a frame near the LC). This means that cosmological redshift and dispersion are not accounted for in these expressions. While redshift is not included in this paper, new smaller dispersion measures are computed using the intrinsic time delay between emitted frequencies given by our model which traces out the magnetic field strength of the LC. These power and flux equations can of course both be integrated with respect to time to compute the total energy and the fluence respectively. 


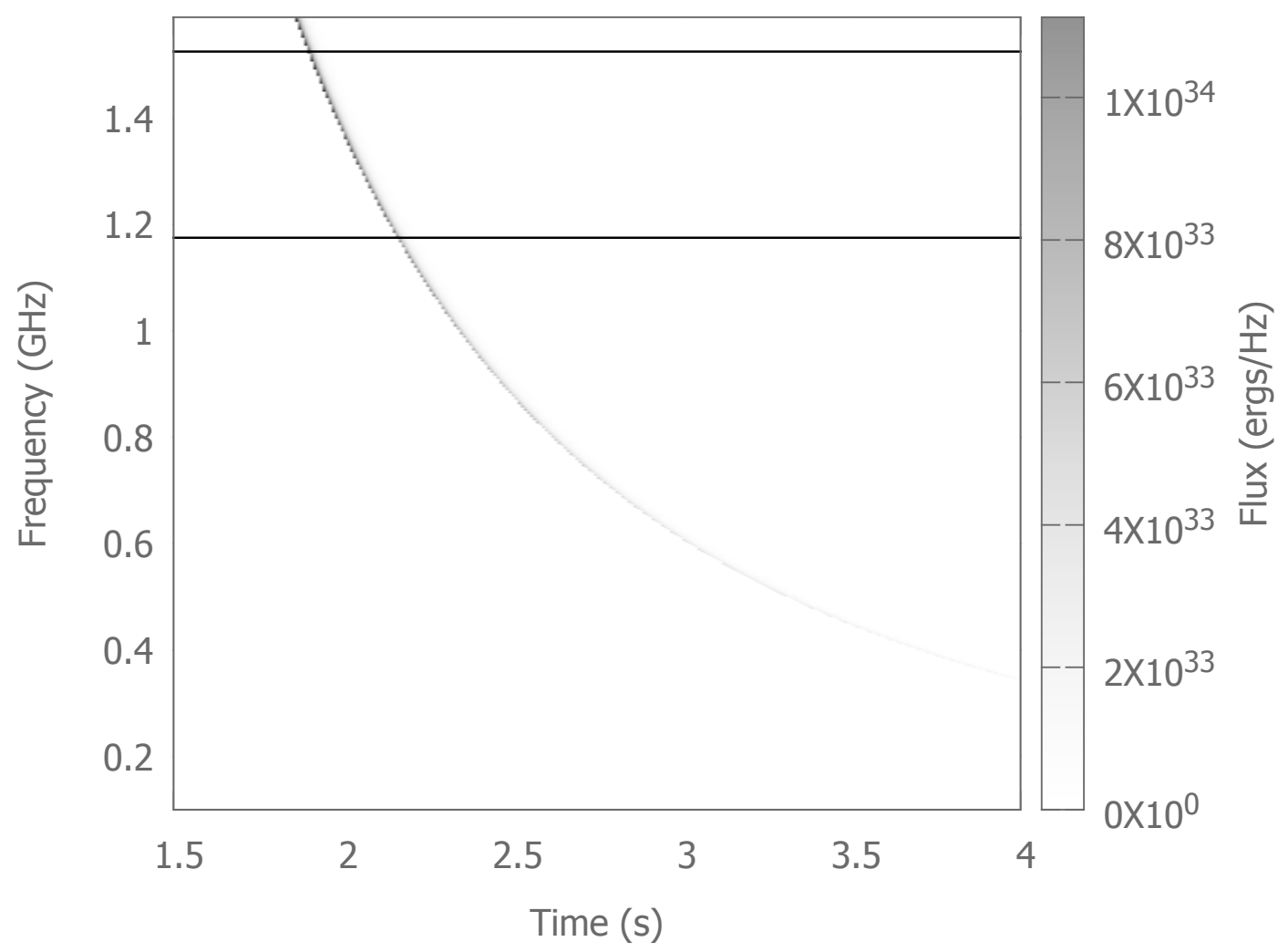

Fig. 6 A spectrograph of the flux density at the emission location. The upper edge of the spectrograph is a cut-off frequency determined by the transition angle of the magnetic field where the magnetic field becomes mono-polar $\left(\phi=30^{\circ}\right)$. The two horizontal lines indicate the bandwidth which has been measured by (Thornton et al. 2013). This plot is a fit to data using the parameters outlined in Table 1 (no statistical fitting was performed to find best fits to the data).

\subsection{Model Assumptions and Limitations}

The model has two major assumptions: 1) the high density ejecta from the QN maintains a distribution of unstable nuclei for far longer than is normally thought possible and 2) the interaction at the LC causes a disruption of the r-process distribution. The r-process stationary distribution and its lifetime have been simulated using the nucleosynthesis code r-Java $2.0^{2}$. While it is a surprising result, we have theoretical background and simulation results to show that it is theoretically possible. The second assumption is much more difficult to quantify. To our knowledge, there has been no theoretical work done on the behaviour of dense nuclear material as it passes at relativistic velocities across a LC. This would require complex magnetohydrodynamic modelling of material near what is already a difficult numerical problem. In order for the model to work, we require a disruption of the r-process distribution at the LC. The most straightforward way to accomplish this is with rapid decompression of the material as shown

\footnotetext{
2 r-Java 2.0 is an open use nucleosynthesis code developed at the University of Calgary at quarknova.ca (Kostka et al. 2014b)
} 


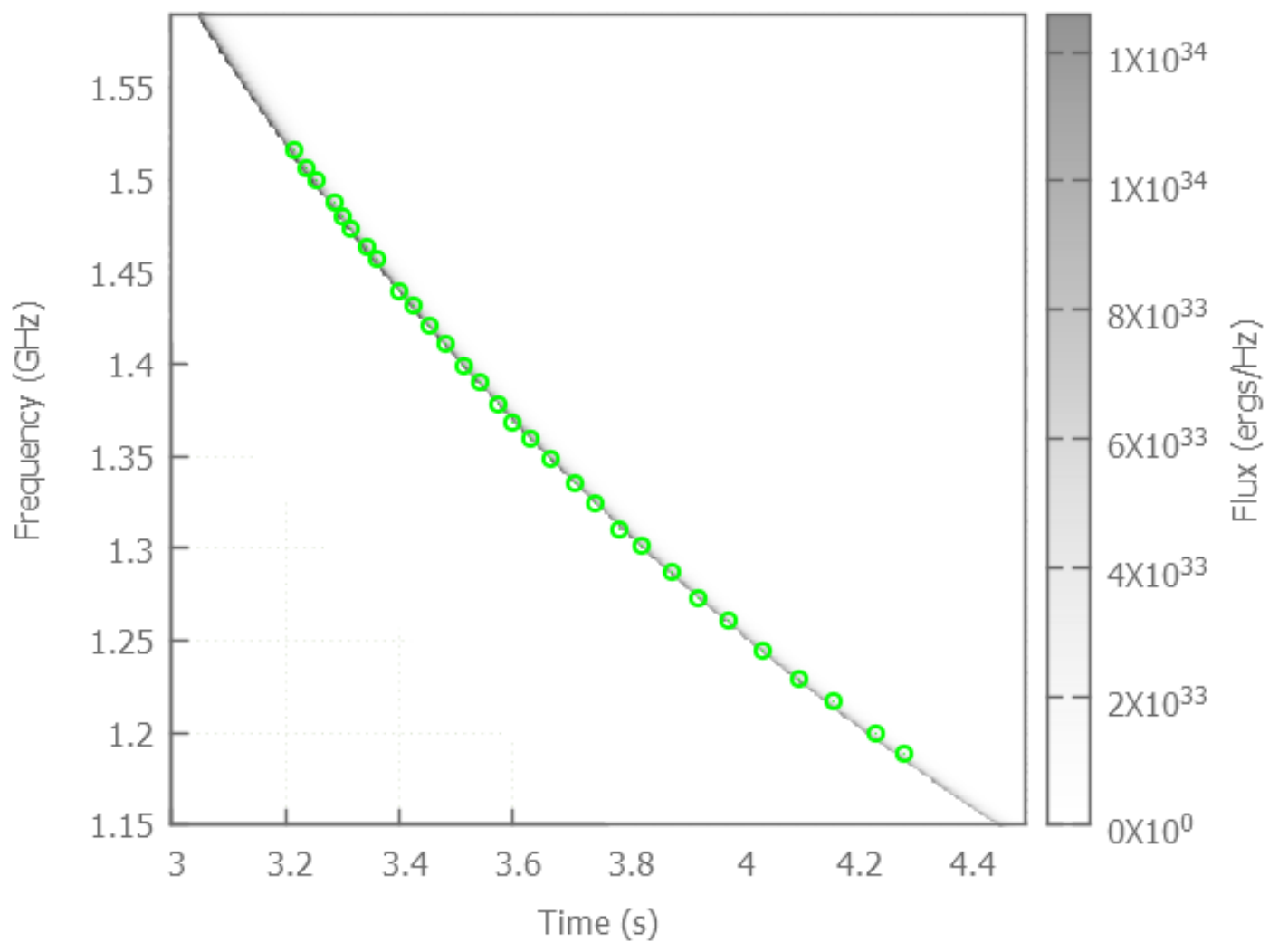

Fig. 7 A spectrograph of the measured data (green points) with our theoretical model predictions. A dispersion measure (DM) of $725 \mathrm{~cm}^{-3} \mathrm{pc}$ has been applied to account for the time delay discrepancy between the emission at source and measurement. The green circles on the plot show where the observational data peaks in the spectrograph.

\begin{tabular}{ll} 
Parameter & Value used \\
\hline$B_{0}$ & $10^{12} \mathrm{G}$ \\
$\phi_{\text {cut }}$ & $30^{\circ}$ \\
$\mathrm{P}$ & $10 \mathrm{~s}$ \\
$\left\{\lambda_{i}\right\}$ & $\{1004,855,597,425,320\} \mathrm{s}^{-1}$ \\
$\beta$ & 0.99 \\
$\gamma$ & 2 \\
$r_{0}$ & $10 \mathrm{~km}$ \\
$m_{\text {QN }, \text { ejecta }}$ & $10^{-3} \mathrm{M}_{\odot}$ \\
$A_{\text {avg }}$ & 180
\end{tabular}

Table 1 Summary of parameter values used in the Fig. 6 and Fig. 8.

quantitatively by the following equation:

$$
r_{(n, \gamma)}=n_{n}\langle\sigma\rangle Y_{i}
$$




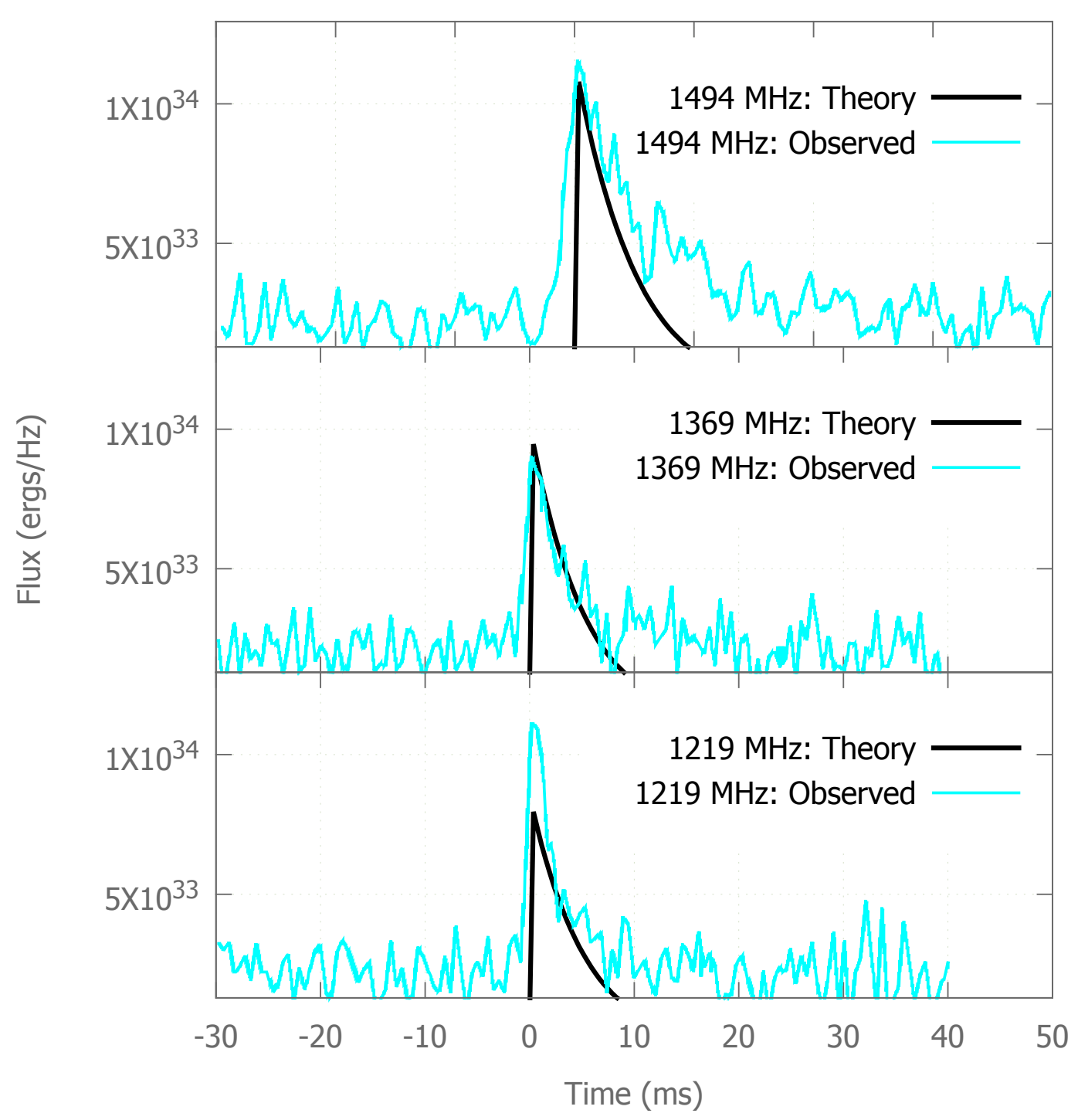

Fig. 8 Slices into the spectrograph of Fig. 6 at 1.494, 1.369 and $1.219 \mathrm{GHz}$ (from left to right). The relative height (or flatness) of the flux at these frequencies across the observing band provides a constraint on the period of the neutron star progenitor. This plot is a fit to data using the parameters outlined in Table 1 (no statistical fitting was performed to find best fits to the data).

where $r_{(n, \gamma)}$ is the neutron capture rate of isotope i, $n_{n}$ is the neutron number density, $\langle\sigma\rangle$ is the average cross section and $Y_{i}$ is the number of isotopes in the system. If the ejecta is rapidly decompressed at the LC this would lead to a rapid decay of all isotopes to stable isotopes.

Other approximations include: a symmetric spherical thin shell expansion of the ejecta, simplified electron energy distributions and use of theoretical decay rates, simplification of the 


$$
\begin{aligned}
& P\left(t=t_{\text {arr }}, \theta, \phi\right)=\oiint_{S(\theta, \phi)} \frac{\partial}{\partial \phi}\left[\Theta(t) \kappa_{p} \gamma_{\perp}^{2} B_{N S}^{2}\left(\frac{2 \pi r_{N S} \cos (\phi)}{c P}\right)^{2 a} \sum_{i}^{\text {chain }}\left(\frac{\mathscr{A}_{n}(t)}{\lambda_{n}}\right)\left(\sin \phi-\sin \phi_{1}\right)\right] d \Omega \\
& S\left(t=t_{\text {arr }}, \theta, \phi\right)=\oiint_{S(\theta, \phi)} \frac{\partial}{\partial \phi}\left[\Theta(t) \frac{\kappa_{p}}{\kappa_{\nu}} B_{0}\left(\frac{2 \pi r_{0} \cos (\phi)}{c P}\right) \sum_{i}^{a \text { chain }}\left(\frac{\mathscr{A}_{n}(t)}{\lambda_{n}}\right)\left(\sin \phi-\sin \phi_{1}\right)\right] d \Omega(15)
\end{aligned}
$$

Fig. 9 Expanded expressions detailing the functional form of the integrands describing the emission spectrum of the QN FRB model. The expression are left unevaluated as derivatives because they become lengthy and do not add any intuition to the problem.

synchrotron radiation spectrum of an electron, and a simplified selection for $\beta$-decay rates. These are each worth exploring in detail to provide a more accurate model; however, the agreement with observations leads us to conclude that these assumptions are all appropriate and physically valid.

\section{RESULTS}

Application of the model to the radio burst FRB 110220-the most spectroscopically complete FRB in Thornton et al. (2013)-is presented in Figs. 6, 7, and 8 using model parameters summarized in Table 1 . The total spectrum emitted using these parameters is presented in Fig. 6. The emission has a maximum frequency and decays for the lower frequencies over time. A comparison to the measured data of FRB 110220 (after a newly inferred dispersion measure is applied) is shown in Figs. 7 and 8.

\subsection{Dispersion Measure, Burst Profile and Distance}

The geometry and fields provide inherent cut-off ranges and characteristic emission timescales for our emitted spectra. The sudden turn-on (as represented by our Heaviside function) at the LC provides us with a frequency leading edge which depends on magnetic field strength, period and - to a lesser extent - magnetic field configuration. The leading edge of the pulse for each frequency (as seen in a spectrograph) is a direct consequence of the geometry. The curve traced out in frequency over time is given by:

$$
\nu_{\text {edge }}=\kappa_{\nu} \gamma^{2} B_{0}\left(\frac{r_{0}}{\beta c}\right)^{a} \frac{1}{t^{a}},
$$

where parameter $a$ is the magnetic field configuration power. Using this, we get a time delay at the source between two frequencies. This can be combined with observations to calculate a dispersion measure of the pulse which will be smaller than that calculated assuming synchronous emission. For FRB 110220 this leads to a DM of $725 \mathrm{~cm}^{-3}$ pc as fit in Fig. 7 which is reduction from $944 \mathrm{~cm}^{-3}$ pc as measured in Thornton et al. (2013).

There is an ambiguity in fitting a dispersion measure from the pulse time which is resolved by the magnetic field strength and the breakpoint between dipole and monopole field lines. 
Since the radiation emitted at the equatorial region is so much fainter, the maximum frequency of the measured spectra is given by:

$$
\nu_{\max }=\kappa_{\nu} \gamma^{2} B_{0}\left(\frac{2 \pi r_{0} \cos \phi_{c u t}}{c P}\right)^{2}
$$

This can be used to help infer the correct dispersion measure of the measured radio burst. This, in combination with the spectral flux at each frequency can be used to calculate a more accurate dispersion measure and distance. The $\mathrm{P}^{-2}$ dependence in Eq. 18 leads to a wide range of possible frequencies. For example, given a millisecond period neutron star $(\mathrm{P}=1 \mathrm{~ms}$, $\mathrm{B}=10^{14} \mathrm{G}$ ) the peak frequency would be $16 \times 10^{18} \mathrm{~Hz}$ putting it in the X-ray regime. This extreme case is unlikely to be realized, as discussed in Section 1. More realistic parameters suggest that FRB-like spectra are present in the optical and infrared bands, which depends on the period and magnetic field strength of FRB progenitors. Determination of the likelihood of emission at different frequencies from this mechanism would require detailed neutron star population studies aimed specifically at determining FRB-QN rates.

In tandem with the new dispersion measure, we can estimate the distance to these QNeFRBs by integrating the flux density or power spectra and comparing this to the measured energy or fluence. This is possible even for bandwidth limited measurements provided there is an estimate from the above quantities of the period and magnetic field by integrating only over the same range of frequencies present in the measurement. If these bursts are in fact cosmological, the cosmological redshift will have to be incorporated into the above equations.

\subsection{FRB Rates}

Assuming that FRB progenitors are old neutron stars, we can estimate their rate based on a simplified population model. We give a rough estimate of the FRB rate by making use of the following assumptions: a constant core-collapse supernova rate $\left(0.02 \mathrm{yr}^{-1} \mathrm{galaxy}^{-1}\right)$, a $\operatorname{lognormal~magnetic~field~distribution~}(\chi(\log B)=13.43 \pm 0.76)$ and a normal distribution for the initial period $\left(\pi\left(\mathrm{P}_{0}\right)=290 \pm 100 \mathrm{~ms}\right)$, where the values for the initial period and magnetic field are taken from Regimbau \& de Freitas Pacheco (2001) (the magnetic field distribution is from the "unseen" population of Model A). In order to decide the lifetime of the neutron stars when they go FRB we've made two choices: 1) they go QN-FRB after a common timescale (or age), or 2) they go QN-FRB once they reach a certain location in the Pि diagram. For the second case, we consider neutron stars reaching critical density near the observed death line and employ the death line of Zhang et al. (2000):

$$
\log \left(\frac{\dot{P}}{\mathrm{~s} \mathrm{~s}^{-1}}\right)=2 \log \left(\frac{P}{\mathrm{~s}}\right)-16.52 .
$$

After modelling the neutron star population of a Milky-Way-like galaxy, the last step is to estimate the fraction of neutron stars which are QN candidates. Since only slow rotating, massive neutron stars are FRB candidates, we count only the neutron stars with periods greater than $100 \mathrm{~ms}$ and whose stellar progenitors had masses between 20-40 $\mathrm{M}_{\odot}$. Integration of the IMF (Kroupa 2001) and the initial period distribution (assuming the period and magnetic field are independent) gives approximately $14 \%$ of neutron stars as QN candidates. This approach does not take into account any possible viewing selection effects (e.g. threshold observing brightness, viewing angle, beaming, etc.), which may affect the detectability of these events. Both the period and death line estimates of the rate give consistent results of approximately 3 per thousand year per galaxy, as summarized in Table 2, which is consistent with the observed FRB rate. 


\begin{tabular}{ll} 
Critical Period (s) & Rate $\left(\mathbf{y r}^{-1}\right.$ galaxy $\left.^{-1}\right)$ \\
\hline 1 & 0.0028 \\
10 & 0.0027 \\
Death Line & 0.0028
\end{tabular}

Table 2 Summary of FRB rates calculated using a simplistic population synthesis of neutron star population.

\section{MODEL CONSEQUENCES AND PREDICTIONS}

While we can generate excellent fits to currently available FRB data, there are other important observables and consequences that come along with the scenario we have put forth to explain these radio transients. As mentioned earlier, we can use this model to constrain certain physical and astronomical phenomena. The relation to nuclear properties is somewhat complex due to the number of nuclear species present and is best left for more detailed analysis and higher resolution measurements. There are, however, two astronomical puzzles that we believe can be explained assuming this model is in fact correct.

\subsection{Neutron Star Population}

Assuming the model is correct, FRBs provide measurements of the period and magnetic field strength of isolated NS before they undergo a QN. Based on the agreement of the model with FRB 110220 and the spectral consistency of the FRB measurements, it appears that these are neutron stars that undergo QN. These neutron stars have periods of around 10 seconds and magnetic fields of $10^{12}$ Gauss (as determined by our parameter selection and its fit to observation data). This is perhaps puzzling if we look at the so-called PP diagram: there are no observed neutron stars in this region (Lorimer \& Kramer 2012). This empty region of the Pम diagram is beyond what is referred to as the "death line" and we propose an explanation for this: once an isolated neutron star spins down to the 1 to 10 second period and enters the neutron star graveyard, it reaches deconfinement densities at its core and dies via a $\mathrm{QN}$ explosion. This instantaneously moves the neutron star into the $10^{15}$ Gauss regime of the diagram as quark stars generate these high magnetic fields after birth (Iwazaki 2005). Instead of magnetars, we then populate this anomalous region of the Pि diagram with quark stars whose magnetic fields decay not through spin down, but through magnetic field expulsion from the color superconducting core (Niebergal et al. 2010, 2006). This drives the quark star from its new position straight downwards as the star ages and provides an explanation for the observed high magnetic field

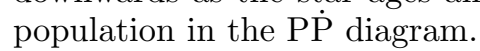

\section{$5.2511 \mathrm{keV}$ Line and Super-heavy Fissionable Elements}

The production of super-heavy $(A>240, Z>92$ ) elements is responsible for the fission recycling in the QN ejecta. While these calculations are based on theoretical predictions of mass models, fission recycling from these elements is theoretically expected. Some of these super-heavy nuclei are also thought to be quasi-stable and long-lived. While they are (currently) impossible to make in the lab, these neutron-rich super-heavy nuclei may outlive the $\beta$-decaying nuclei and undergo fission much later. If this is correct, these super-heavy nuclei would fission and emit photons over a long period of time and produce extended emission of $\mathrm{MeV}$ photons. These emitted photons then have the appropriate energy for efficient production of electron-positron pairs (via $\gamma-\gamma$ annihilation). The annihilation events would produce $511 \mathrm{keV}$ photons when the produced positrons find an electron and provide a potential explanation for the positron 
emission which is an observed phenomenon that still has no definitive explanation for its galactic origin (Weidenspointner et al. 2008).

This radiation would of course not be as energetic as the original FRB and is likely not measurable from an extragalactic source; however, this does provide a possible explanation for the $511 \mathrm{keV}$ photons observed in our own galaxy. The current observed annihilation rate of positrons inferred from the $511 \mathrm{keV}$ positronium line is $\sim 10^{43} \mathrm{~s}^{-1}$ (Knödlseder et al. 2005). Based on our model, we can then constrain the lifetime of super-heavy neutron rich nuclei to be on the order of 1000 years using the following logic.

Given a galactic QN rate of 1 per thousand years, if we assume the following parameters: $M_{\mathrm{QN} \text {,ejected }}=10^{-2} \mathrm{M}_{\odot}$, average nuclei mass $\mathrm{A} \sim 100$ and $10 \%$ of nuclei (by number) are super-heavy fissionable elements, we can expect about $10^{-5} M_{\odot}$ nuclei produced in the QN ejecta which gives about $10^{51}$ fissionable nuclei per QN event. Given the expected QN rate of 0.001 year $^{-1}$ this produces an average $10^{48}$ super-heavy nuclei per year. If we compare this to the current event rate this implies a characteristic lifetime of about 1000 years for the fission events (see Petroff et al. (2015) for further discussion of observational qualities and proposed astrophysical theories).

While these calculations are an order of magnitude approximation, it has several promising features. QNe come from massive $\left(20-40 M_{\odot}\right)$ stars and are expected to operate (like the $\mathrm{r}-$ process) continually over the entire history of the galaxy. This leads naturally to a concentration of $511 \mathrm{keV}$ lines near the active portions of the galaxy (the bulge and the disk) where most of this emission is observed. This matches the observed clustering of the 511 positron emission measured (Weidenspointner et al. 2008). Additionally, because a quark star is an aligned rotator, we expect no radio pulsations to be observed alongside this emission which, again, seems to agree with observation and is one of the difficult aspects in explaining these positronium annihilation events. Finally, since the galactic centre and plane has many high energy photons which would allow for efficient conversion of the $\mathrm{MeV}$ photons from fission to electron-positron pairs (because the pair production requires a $\gamma-\gamma$ interaction). This emission should then carry a P Cygni profile as well as a net exponential decay which could be detected from observations.

It is also possible that the heavy nuclei do not spontaneously fission, but instead undergo photo-fission triggered by the high energy photons present in large numbers especially near the galactic centre. This would lead to a slightly different observable: instead of half-lives we could infer photo-fission cross sections which would give indirect measurements of the level density and fission barrier heights of these nuclei. Instead of a plain exponential, we would instead observe rates proportional to the photon density.

\section{CONCLUSION}

Quark novae are capable of generating fast radio pulses using electrons generated by r-process nuclei. Assuming decompression or any other disruption of the r-process at the neutron star's LC, we have shown how the emission spectrum has an intrinsic time delay across its frequency band and has a predictable bandwidth (cut-off frequency) which can be used to measure magnetic field strength and period of otherwise undetectable neutron stars. If correct, FRBs can be used to probe and measure properties of unstable nuclear matter and measure properties of old neutron star populations. Our model predicts that these explosions are indeed one of the most energetic explosions in radio astronomy (with energies on the order of $10^{41} \mathrm{ergs}$ ); however, they need not necessarily be cosmological as is inferred from previous analysis of the data. The model naturally provides explanations for the timescales and energies of the FRB emissions using synchrotron emission in the strong magnetic field of a birthing quark nova.

\section{References}

Charignon, C., Kostka, M., Koning, N., Jaikumar, P., \& Ouyed, R. 2011, A\&A, 531, A79 
Contopoulos, I., Kazanas, D., \& Fendt, C. 1999, ApJ, 511, 351

Drago, A., Lavagno, A., Pagliara, G., \& Pigato, D. 2015, arXiv:1509.02131

Falcke, H., \& Rezzolla, L. 2014, A\&A, 562, A137

Flores, C. V., \& Lugones, G. 2014, Classical and Quantum Gravity, 31, 155002

Fuller, J., \& Ott, C. D. 2015, MNRAS, 450, L71

Geng, J. J., \& Huang, Y. F. 2015, ApJ, 809, 24

Geng, J. J., Huang, Y. F., \& Lu, T. 2015, ApJ, 804, 21

Gondek-Rosińska, D., Gourgoulhon, E., \& Haensel, P. 2003, A\&A, 412, 777

Goriely, S., Hilaire, S., \& Koning, A. J. 2008, A\&A, 487, 767

Goriely, S., Hilaire, S., \& Koning, A. J. 2009, in American Institute of Physics Conference Series, Vol. 1090, American Institute of Physics Conference Series, ed. J. Jolie, A. Zilges, N. Warr, \& A. Blazhev, 629

Iwazaki, A. 2005, Phys. Rev. D, 72, 114003

Jaikumar, P., Meyer, B. S., Otsuki, K., \& Ouyed, R. 2007, A\&A, 471, 227

Jessner, A., Lesch, H., \& Kunzl, T. 2000, in Astronomical Society of the Pacific Conference Series, Vol. 202, IAU Colloq. 177: Pulsar Astronomy - 2000 and Beyond, ed. M. Kramer, N. Wex, \& R. Wielebinski, 463

Keränen, P., Ouyed, R., \& Jaikumar, P. 2005, ApJ, 618, 485

Knödlseder, J., Jean, P., Lonjou, V., et al. 2005, A\&A, 441, 513

Kostka, M., Koning, N., Leahy, D., Ouyed, R., \& Steffen, W. 2014a, Revista Mexicana de Astronomía y Astrofísica, 50, 167

Kostka, M., Koning, N., Shand, Z., Ouyed, R., \& Jaikumar, P. 2014b, A\&A, 568, A97

Krane, K. S., \& Halliday, D. 1988, Introductory nuclear physics (Wiley)

Krücken, R. 2011, Contemporary Physics, 52, 101

Kroupa, P. 2001, MNRAS, 322, 231

Lang, K. R. 1999, Astrophysical formulae (Springer)

Lorimer, D. R., \& Kramer, M. 2012, Handbook of Pulsar Astronomy (Cambridge University Press)

Lyubarsky, Y. 2014, MNRAS, 442, L9

Michel, F. C. 1973, ApJ, 180, 207

Möller, P., Pfeiffer, B., \& Kratz, K.-L. 2003, Phys. Rev. C, 67, 055802

Niebergal, B., Ouyed, R., \& Leahy, D. 2006, ApJ, 646, L17

Niebergal, B., Ouyed, R., Negreiros, R., \& Weber, F. 2010, Phys. Rev. D, 81, 043005

Ouyed, R., Dey, J., \& Dey, M. 2002, A\&A, 390, L39

Ouyed, R., Koning, N., \& Leahy, D. 2013, Research in Astronomy and Astrophysics, 13, 1463

Ouyed, R., \& Leahy, D. 2009, ApJ, 696, 562

Ouyed, R., Niebergal, B., Dobler, W., \& Leahy, D. 2006, ApJ, 653, 558

Ouyed, R., Niebergal, B., \& Jaikumar, P. 2013, arXiv:1304.8048

Ouyed, R., Staff, J., \& Jaikumar, P. 2011, The Astrophysical Journal, 729, 60

Pen, U.-L., \& Connor, L. 2015, ApJ, 807, 179

Petroff, E., Bailes, M., Barr, E. D., et al. 2015, MNRAS, 447, 246

Popov, S. B., \& Postnov, K. A. 2007, arXiv:0710.2006

Popov, S. B., \& Postnov, K. A. 2013, arXiv:1307.4924

Regimbau, T., \& de Freitas Pacheco, J. A. 2001, A\&A, 374, 182

Spitkovsky, A. 2006, ApJ, 648, L51

Staff, J. E., Ouyed, R., \& Jaikumar, P. 2006, ApJ, 645, L145

Thornton, D., Stappers, B., Bailes, M., et al. 2013, Science, 341, 53

Totani, T. 2013, PASJ, 65, L12

Weidenspointner, G., Skinner, G. K., Jean, P., et al. 2008, New Astron. Rev., 52, 454

Zhang, B., Harding, A. K., \& Muslimov, A. G. 2000, ApJ, 531, L135 